\title{
A NOTE ON CLEBSCH-GORDAN INTEGRAL, FOURIER-LEGENDRE EXPANSIONS AND CLOSED FORM FOR HYPERGEOMETRIC SERIES
}

\author{
MARCO CANTARINI
}

\begin{abstract}
In this paper we show that a closed form formula for the generalized Clebsch-Gordan integral and the Fourier-Legendre expansion theory allow to evaluate hypergeometric series involving powers of the normalized central binomial coefficient $\frac{1}{4^{n}}\left(\begin{array}{c}2 n \\ n\end{array}\right)$.
\end{abstract}

Mathematical Subject Classification 2020: 33C20, 33E05, 42C10, 33C75.

\section{INTRODUCTION}

The study of hypergeometric transformation, and its link to the analysis of closed-form of infinite series in terms of well-known mathematical constants and special values of Euler's Gamma function, has been deeply analyzed in many articles and with different techniques. Indeed, it is well known that this type of research is of interest mathematics and in other scientific fields; a very exhaustive illustration can be found in [5]. Among the many tools developed, recently it was shown that the Fourier-Legendre (FL) expansion theory is a very useful approach for the study of a class of hypergeometric series, in particular series whose summands are powers of the normalized central binomial coefficients, harmonic numbers and rational functions (see [9, [14]) because it allows to formulate these series in terms of Euler sums or in integrals involving special functions like polylogarithms or complete elliptic integrals of the first and second kind. This information shows again the interest about this type of problems since, as we know, the evaluation of multiple elliptic integrals where the integrands are combinations of complete elliptic integrals of the first or second kind is an active research area and with applications in, for example, high-energy physics, statistical mechanics and probability theory. Very recently [10] it was observed that the FL theory combined with the theory of fractional operators, in particular with semi-differentiation and semi-integration (for some details about fractional calculus see, for example, [18]) of scalar product of some functions, including complete elliptic integrals of the first or second kind, allow us to evaluate particular hypergeometric functions with fractional (in particular, quarter-integers) parameters.

It is important to emphasize again how these topics and techniques can be relate to other, and sometimes unexpected, mathematical topics; for example, if it is quite natural to think about the classical Ramanujan-type series for $1 / \pi$ (for a survey of this topic see for example, [3] and for formulas via hypergeometric transformations see [15]), the connection with additive number theory problems is probably less evident, in particular asymptotic formulas of functions that count the number of representations of an integer as the sum of elements that are in some subset of natural numbers (essentially, primes or powers of primes). Indeed, fractional operators applied to particular power series are involved in the study of explicit formulas for the so-called Cesàro average of these counting functions (for the interested reader, see [7] [11] [12] [13] 20]), therefore it is plausible to think that the techniques developed may also be of interest for these types of problems In this paper, we will focus on a results of Zhou [21] about a closed form for the generalized Clebsch-Gordan integral

$$
\int_{-1}^{1} P_{\mu}(x) P_{\nu}(x) P_{\nu}(-x) d x
$$

Key words and phrases. Hypergeometric functions, Fourier-Legendre expansion, Clebsch-Gordan integral, complete elliptic integral of the first kind, closed form. 
where $P_{\nu}(x), P_{\mu}(x)$ are the Legendre functions of arbitrary complex degree $\nu, \mu \in \mathbb{C}$. We show that this result can be can be interpreted in terms of the FL theory and this point of view allows to evaluate series whose addends are powers of central binomials (and so, particular hypergoemetric functions). Furthermore, we will show that from Zhou results we can obtain some formulas that recall the well-known Brafman's formula [6] and we can evaluate very easily some integral moment regarding combinations of complete elliptic integrals of the first kind.

Note that we adopt the convention whereby the argument of a complete elliptic integral is the elliptic modulus, that is

$$
K(x):=\int_{0}^{\pi / 2} \frac{d u}{\sqrt{1-x \sin ^{2}(u)}} .
$$

I thank the referee very much for the comments and suggestions, which have greatly improved the article.

\section{On some CONSEQUences of Zhou's PaPer}

We start our analysis observing that the closed form for the Clebsch-Gordan integral can be interpreted as the FL expansions of a combination of particular Gauss hypergeometric functions ${ }_{2} F_{1}(a, b ; c ; z)$.

Theorem 1. Let $\nu \in \mathbb{C}$ and $x \in[0,1]$. The following $F L$ expansions there holds:

$$
\begin{gathered}
{ }_{2} F_{1}(-\nu, \nu+1 ; 1 ; x){ }_{2} F_{1}(-\nu, \nu+1 ; 1 ; 1-x) \\
=-\frac{\sin (\pi \nu)}{2} \sum_{m \geq 0}\left[\frac{1}{4^{m}}\left(\begin{array}{c}
2 m \\
m
\end{array}\right)\right]^{2} \frac{\Gamma(m-\nu) \Gamma(m+\nu+1)(4 m+1)}{\Gamma\left(m-\nu+\frac{1}{2}\right) \Gamma\left(m+\nu+\frac{3}{2}\right)} P_{2 m}(2 x-1)
\end{gathered}
$$

where the indeterminate form must be interpreted as limits.

Proof. Recalling that

$$
{ }_{2} F_{1}(-\nu, \nu+1 ; 1 ; x)=P_{\nu}(1-2 x), x \in[0,1], \nu \in \mathbb{C},
$$

(see equation 6.2 of [19]) we have, for $m \in \mathbb{N}$,

$$
\begin{aligned}
& \int_{0}^{1}{ }_{2} F_{1}(-\nu, \nu+1 ; 1 ; x){ }_{2} F_{1}(-\nu, \nu+1 ; 1 ; 1-x) P_{m}(2 x-1) d x \\
& =\int_{0}^{1} P_{\nu}(1-2 x) P_{\nu}(2 x-1) P_{m}(2 x-1) d x \\
& =\int_{-1}^{1} P_{\nu}(x) P_{\nu}(-x) P_{m}(x) d x .
\end{aligned}
$$

From equation $\left(19_{(m, n)}\right)$ and $\left(19_{(2 m+1, \nu)}\right)$ of [21] we have that (2) is 0 for any Legendre polynomial $P_{n}(2 x-1)$ of odd degree $n$ and

$$
\int_{-1}^{1} P_{\nu}(x) P_{\nu}(-x) P_{2 m}(x) d x=-\frac{\sin (\pi \nu)}{2}\left[\frac{1}{4^{m}}\left(\begin{array}{c}
2 m \\
m
\end{array}\right)\right]^{2} \frac{\Gamma(m-\nu) \Gamma(m+\nu+1)}{\Gamma\left(m-\nu+\frac{1}{2}\right) \Gamma\left(m+\nu+\frac{3}{2}\right)}
$$

where $m$ is a positive integer, $\nu \in \mathbb{C}$ and the indeterminate form must be interpreted as limits. The thesis follows recalling that if $f(x) / \sqrt[4]{1-x^{2}}, x \in(-1,1)$ is integrable, then

$$
\sum_{n \geq 0}\left(n+\frac{1}{2}\right) P_{n}(\xi) \int_{-1}^{1} f(x) P_{n}(x) d x=\frac{f(\xi+0)-f(\xi-0)}{2}
$$

for a certain $\xi \in(-1,1)$ if some conditions for convergence are met (for more details see [17, Chapter VII, p. 329).

As we had anticipated, the previous formula clearly recalls the well-known Brafman's formula (see [6]) and it has some interesting consequences. 
Theorem 2. For $\nu \in \mathbb{C} \backslash(\{-2 \mathbb{N}+1\} \cup\{2 \mathbb{N}\})$ we have

$$
\frac{\cot \left(\frac{\pi \nu}{2}\right) \Gamma\left(\frac{1+\nu}{2}\right)^{2}}{\pi \Gamma\left(\frac{2+\nu}{2}\right)^{2}}=\sum_{m \geq 0}\left[\frac{1}{4^{m}}\left(\begin{array}{c}
2 m \\
m
\end{array}\right)\right]^{3}(-1)^{m+1} \frac{\Gamma(m-\nu) \Gamma(m+\nu+1)(4 m+1)}{\Gamma\left(m-\nu+\frac{1}{2}\right) \Gamma\left(m+\nu+\frac{3}{2}\right)}
$$

where the undetermined forms must be interpreted as limits and, for $x \in[0,1]$, we have

$$
K(x) K(1-x)=\frac{\pi^{3}}{8} \sum_{m \geq 0}\left[\frac{1}{4^{m}}\left(\begin{array}{c}
2 m \\
m
\end{array}\right)\right]^{4}(4 m+1) P_{2 m}(2 x-1) .
$$

Proof. Due to the fact that

$$
{ }_{2} F_{1}\left(-\nu, \nu+1 ; 1 ; \frac{1}{2}\right)=\frac{\sqrt{\pi}}{\Gamma\left(\frac{1-\nu}{2}\right) \Gamma\left(\frac{\nu+2}{2}\right)}
$$

by the Gauss's second summation theorem [2], formula (3) follows from the $x=1 / 2$ case of (11) and (4) follows from the $\nu=-1 / 2$ case of (1).

These results produce some interesting identities that CAS like Mathematica does not recognize or it only recognizes them as combinations of generalized hypergeometric functions; in the next corollary we show some interesting examples. Note that we will write the combination of hypergeometric functions only in cases where the CAS is able to recognize it and if the formula contains at most two terms, to make the results more readable.

Corollary 3. We have

$$
\begin{aligned}
\sum_{m \geq 0}\left[\frac{1}{4^{m}}\left(\begin{array}{c}
2 m \\
m
\end{array}\right)\right]^{5}(-1)^{m}(4 m+1) & \left(\psi^{(1)}\left(m+\frac{1}{2}\right)-\psi^{(1)}(m+1)\right)=\frac{2 \Gamma\left(\frac{1}{4}\right)^{4} C}{\pi^{4}} \\
\sum_{m \geq 0}\left[\frac{1}{4^{m}}\left(\begin{array}{c}
2 m \\
m
\end{array}\right)\right]^{5}(-1)^{m}(4 m+1) & =\frac{1}{8}\left(8 F_{4}\left(\frac{1}{2}, \frac{1}{2}, \frac{1}{2}, \frac{1}{2}, \frac{1}{2} ; 1,1,1,1 ;-1\right)\right. \\
& \left.-{ }_{5} F_{4}\left(\frac{3}{2}, \frac{3}{2}, \frac{3}{2}, \frac{3}{2}, \frac{3}{2} ; 2,2,2,2 ;-1\right)\right) \\
& =\frac{\Gamma\left(\frac{1}{4}\right)^{4}}{2 \pi^{4}}
\end{aligned}
$$

$$
\sum_{m \geq 0}\left[\frac{1}{4^{m}}\left(\begin{array}{c}
2 m \\
m
\end{array}\right)\right]^{3} \frac{(-1)^{m+1}(4 m-1)(4 m+3)}{(4 m-3)(4 m+5)}=\frac{32 \sqrt{2}(1+\sqrt{2}) \Gamma\left(\frac{1}{4}\right)^{2}}{9 \Gamma\left(\frac{1}{8}\right)^{4}}
$$$$
\sum_{m \geq 0}\left[\frac{1}{4^{m}}\left(\begin{array}{c}
2 m \\
m
\end{array}\right)\right]^{5} \frac{(-1)^{m}(4 m+1)\left(4 m^{2}+2 m+1\right)}{(2 m-1)^{2}(m+1)^{2}}=\frac{128}{\Gamma\left(\frac{1}{4}\right)^{4}}
$$

where $C$ is the Catalan's constant and $\psi^{(1)}(x)$ is the trigamma function.

Proof. Equations (6) and (7) follow from the $\nu=1 / 4$ and $\nu=3 / 4$ cases of (3); differentiating (8) with respect to $\nu$ before specializing to $\nu=1 / 2$, one arrives to (6); twice differentiating (6) with respect to $\nu$ before specializing $\nu=-1 / 2$, one arrives to (9); equation (10) follows from the $x=1 / 2$ case of (4).

Note that formula (4) allow us to evaluate quite easily the moments of the function $K(x) K(1-x)$. 
Corollary 4. For every $n \in \mathbb{N}$ we have

$$
\begin{aligned}
& \int_{0}^{1} x^{n} K(x) K(1-x) d x \\
& =\frac{\pi^{3} \Gamma(n+1)^{2}}{8} \sum_{m \leq n / 2}\left[\frac{1}{4^{m}}\left(\begin{array}{c}
2 m \\
m
\end{array}\right)\right]^{4} \frac{4 m+1}{\Gamma(n+2 m+2) \Gamma(n+1-2 m)}
\end{aligned}
$$

and for every $n \in \mathbb{N}^{+}$we have

$$
\begin{aligned}
& \int_{0}^{1} x^{n-1} K(x)(1-x)^{n-1} K(1-x) d x \\
& =\frac{\pi^{7 / 2}}{8} \frac{\Gamma(n)^{3} \Gamma\left(n+\frac{1}{2}\right)}{\Gamma(2 n)} \sum_{m<n}\left[\frac{1}{4^{m}}\left(\begin{array}{c}
2 m \\
m
\end{array}\right)\right]^{4} \frac{4 m+1}{\Gamma\left(\frac{1-2 m}{2}\right) \Gamma(m+1) \Gamma\left(\frac{2 m+1}{2}+n\right) \Gamma(-m+n)} .
\end{aligned}
$$

Proof. Using (4) and switching the integral with the series (it is quite easy to prove that it is allowed) we get

$$
\int_{0}^{1} x^{n} K(x) K(1-x) d x=\frac{\pi^{3}}{8} \sum_{m \geq 0}\left[\frac{1}{4^{m}}\left(\begin{array}{c}
2 m \\
m
\end{array}\right)\right]^{4}(4 m+1) \int_{0}^{1} x^{n} P_{2 m}(2 x-1) d x
$$

and the result follows by the well-known identity

$$
\int_{0}^{1} x^{\mu-1} P_{n}(2 x-1) d x=\frac{\Gamma(\mu)^{2}}{\Gamma(\mu+n+1) \Gamma(\mu-n)}, \operatorname{Re}(\mu)>0
$$

(see [16], page 792). Similarly, for the second identity we use the relation

$$
\begin{aligned}
& \int_{0}^{1} x^{\mu-1}(1-x)^{\nu-1} P_{n}(2 x-1) d x \\
& =(-1)^{n} \frac{\Gamma(\mu) \Gamma(\nu)}{\Gamma(\nu+\mu)}{ }_{3} F_{2}(-n . n+1, \mu ; 1, \mu+\nu ; 1), \operatorname{Re}(\mu)>0, \operatorname{Re}(\nu)>0
\end{aligned}
$$

(see [16, page 792) and the classical Watson theorem (see, for example, [1], Theorem 3.5.5).

$$
{ }_{3} F_{2}\left(a, b, c ; \frac{a+b+1}{2}, 2 c ; 1\right)=\frac{\sqrt{\pi} \Gamma\left(c+\frac{1}{2}\right) \Gamma\left(\frac{a+b+1}{2}\right) \Gamma\left(\frac{1-a-b}{2}+c\right)}{\Gamma\left(\frac{a+1}{2}\right) \Gamma\left(\frac{b+1}{2}\right) \Gamma\left(\frac{1-a}{2}+c\right) \Gamma\left(\frac{1-b}{2}+c\right)},
$$

with $\operatorname{Re}(-a-b+2 c)>-1$

\section{Dougall's expansions, Mehler-Dirichlet theory AND Fl EXPASions}

There are interesting applications of Lemma 2.1 in Zhou's paper, when we combine it with other identities. We recall the Dougall's expansion (see 4, page 167)

$$
{ }_{2} F_{1}(-\nu, \nu+1 ; 1 ; x)=\sum_{m \geq 0}\left[\frac{\sin (\pi(m-\nu))}{\pi(m-\nu)}+\frac{\sin (\pi(m+\nu+1))}{\pi(m+\nu+1)}\right] P_{m}(2 x-1)
$$

where the indeterminate forms must be interpreted as limits. Clearly, this identity can be read as the FL expansion of the function ${ }_{2} F_{1}(-\nu, \nu+1 ; 1 ; x)$.

Corollary 5. We have that

$$
\begin{aligned}
\sum_{m \geq 0}\left(\begin{array}{c}
2 m \\
m
\end{array}\right) \frac{(-1)^{m}}{4^{m}}\left[\frac{1}{(4 m-1)^{2}}-\frac{1}{(4 m+3)^{2}}\right] & =\frac{1}{9}\left(9{ }_{3} F_{2}\left(-\frac{1}{4},-\frac{1}{4}, \frac{1}{2} ; \frac{3}{4}, \frac{3}{4} ;-1\right)\right. \\
& \left.-{ }_{3} F_{2}\left(\frac{1}{2}, \frac{3}{4}, \frac{3}{4} ; \frac{7}{4}, \frac{7}{4} ;-1\right)\right) \\
& =\frac{2 \pi^{3 / 2}}{\Gamma\left(\frac{1}{4}\right)^{2}} .
\end{aligned}
$$


Proof. From (5) and (11) we get, taking $x=1 / 2$ and recalling the well known relation

$$
P_{m}(0)= \begin{cases}\left(\begin{array}{c}
2 m \\
m
\end{array}\right) \frac{(-1)^{m}}{4^{m}}, & m \text { even } \\
0, & m \text { odd }\end{cases}
$$

that

$$
\frac{\sqrt{\pi}}{\Gamma\left(\frac{1-\nu}{2}\right) \Gamma\left(\frac{\nu+2}{2}\right)}=\sum_{m \geq 0}\left(\begin{array}{c}
2 m \\
m
\end{array}\right) \frac{(-1)^{m}}{4^{m}}\left[\frac{\sin (\pi(2 m-\nu))}{\pi(2 m-\nu)}+\frac{\sin (\pi(2 m+\nu+1))}{\pi(2 m+\nu+1)}\right]
$$

and now the claim follows differentiating with respect $\nu$ both sides and then taking $\nu=$ $1 / 2$.

Note that this result is interesting because, despite the seemingly simple appearance, series like (12) are often linked to known, and important, mathematical constants but could be, in general, difficult to deal with. An example is the series

$$
\sum_{m \geq 0}\left(\begin{array}{c}
2 m \\
m
\end{array}\right) \frac{(-1)^{m}}{4^{m}} \frac{1}{(4 m+1)^{2}}
$$

which is closely related to the series

$$
\sum_{m \geq 0}\left(\begin{array}{c}
2 m \\
m
\end{array}\right) \frac{1}{4^{m}} \frac{H_{m}}{4 m+1}
$$

and both are linked to lemnistate-like constants but, at present, no technique is known for calculating their closed forms (see, for more details on this topic, [8]).

Other interesting relations can be extrapolated from Zhou's paper; indeed, from the wellknown FL expansion

$$
K(x)=\sum_{m \geq 0} \frac{2}{2 m+1} P_{m}(2 x-1), x \in[0,1),
$$

the Mehler-Dirichlet theory, the Hobson coupling formula, which states that for $\nu \in \mathbb{C}$ and $\theta_{1}, \theta_{2} \in[0, \pi)$ we have

$$
\begin{aligned}
& \frac{1}{2 \pi} \int_{0}^{2 \pi} P_{\nu}\left(\cos \left(\theta_{1}\right) \cos \left(\theta_{2}\right)+\sin \left(\theta_{2}\right) \sin \left(\theta_{2}\right) \cos (\phi)\right) d \phi \\
& = \begin{cases}P_{\nu}\left(\cos \left(\theta_{1}\right)\right) P_{\nu}\left(\cos \left(\theta_{2}\right)\right), & \theta_{1}+\theta_{2} \leq \pi \\
P_{\nu}\left(-\cos \left(\theta_{1}\right)\right) P_{\nu}\left(-\cos \left(\theta_{2}\right)\right), & \theta_{1}+\theta_{2} \geq \pi\end{cases}
\end{aligned}
$$

and its consequences (see Lemma 2.1 of [21]), it is possible to obtain the following "quasi" FL- expansions

$$
\begin{gathered}
\sum_{m \geq 0} \frac{P_{m}(2 x-1)^{2}(-1)^{m}}{2 m+1}=\frac{K(x)^{2}}{\pi}, x \in[0,1 / 2], \\
\sum_{m \geq 0} P_{m}(2 x-1)^{2} z^{n}=\frac{2}{\pi} \frac{K\left(-\frac{16 x(1-x) z}{(1-z)^{2}}\right)}{1-z}, x, z \in(0,1)
\end{gathered}
$$

and then we are able to find the following identities:

Corollary 6. We have that

$$
\begin{gathered}
\frac{2}{\pi} \int_{0}^{1} \frac{K\left(\frac{16 x(1-x) z^{2}}{\left(1+z^{2}\right)^{2}}\right)}{1+z^{2}} d z= \begin{cases}\frac{K(x)^{2}}{\pi}, & x \in[0,1 / 2] \\
\frac{K(1-x)^{2}}{\pi}, & x \in[1 / 2,1]\end{cases} \\
\frac{2}{\pi} \int_{0}^{1} \frac{K\left(\frac{16 x(1-x) z^{2}}{\left(1+z^{2}\right)^{2}}\right)}{1+z^{2}} d x=\frac{\arctan (z)}{z}, z \in(0,1) .
\end{gathered}
$$




\section{Conclusions}

We have shown some examples of how FL theory is a useful tool for dealing with computational problems linked to some types of hypergeometric functions and how its flexibility allows, at least in the first instance, to be exploited in other areas of mathematics. We want to underline how the results presented in this work are only a part of the possible ones obtainable from the general formulas and how these techniques could be used in other fields of mathematics and beyond; this lead us to continue our investigation on these topics and we hope to produce other interesting results in the future.

\section{Acknowledgments}

The author is a member of the Gruppo Nazionale per l'Analisi Matematica, la Probabilità e le loro Applicazioni (GNAMPA) of the Istituto Nazionale di Alta Matematica (INdAM).

\section{REFERENCES}

[1] G. E. Andrews, R. Askey and R. Roy, Special Functions, volume 71 of Encyclopedia of Mathematics and Its Applications. Cambridge University Press, Cambridge, UK, 1999.

[2] W. N. Bailey, Generalized Hypergeometric Series, Cambridge University Press, Cambridge, 1935.

[3] N. D. Baruah, B. C. Berndt, H. H. Chan, Ramanujan's series for $1 / \pi$ : a survey, Amer. Math. Monthly 116 (2009), 567-587

[4] H. Bateman, Higher Transcendental Functions, volume I, McGraw-Hill, New York, NY, 1953. (compiled by staff of the Bateman Manuscript Project: Arthur Erdélyi, Wilhelm Magnus, Fritz Oberhettinger, Francesco G. Tricomi, David Bertin, W. B. Fulks, A. R. Harvey, D. L. Thomsen, Jr., Maria A. Weber and E. L. Whitney).

[5] J. M. Borwein, R. E. Crandall, Closed forms: what they are and why we care, Notices Amer Math Soc. 60 (1) (2013), 50-65.

[6] F. Brafman, Generating functions of Jacobi and related polynomials, Proc. of the American Math. Soc. 2 (6) (1951) 942-949.

[7] J. Brüdern, J. Kaczorowski, and A. Perelli, Explicit formulae for averages of Goldbach representations, Trans. Amer. Math. Soc. 372 (2019), 6981-6999.

[8] J. M. Campbell, W. Chu, Lemniscate-like constants and infinite series, accepted by Mathematica Slovaca.

[9] J.M. Campbell, J. D'Aurizio, J. Sondow. On the interplay among hypergeometric functions, complete elliptic integrals, and Fourier-Legendre expansions, J. Math. Anal. Appl., 479(1) (2019), 90-121.

[10] J. M. Campbell, M. Cantarini, J. D'Aurizio, Symbolic computations via Fourier-Legendre expansions and fractional operators, accepted by Integral Transforms and Special Functions, https://doi.org/10.1080/10652469.2021.1919103.

[11] M. Cantarini, On the Cesàro average of the 'Linnik numbers'. Acta Arith. 180(1) (2017), 45-62.

[12] M. Cantarini, On the Cesàro average of the numbers that can be written as sum of a prime and two squares of primes, Journal of Number Theory 185 (2018),194-217.

[13] M. Cantarini, Some identities involving the Cesàro average of the Goldbach numbers, Math. Notes 106(5-6) (2019), 688-702.

[14] M. Cantarini, J. D'Aurizio, On the interplay between hypergeometric series, Fourier-Legendre expansions and Euler sum, Bollettino Unione Matematica Italiana 12(4) (2019), 623-656.

[15] S. Cooper, J. Ge, D. Ye, Hypergeometric transformation formulas of degrees 3, 7, 11 and 23, J. Math. Anal. Appl. 421(2) (2015), 1358-1376.

[16] I. S. Gradshteyn and I. M. Ryzhik, Table of Integrals, Series, and Products, edited by A. Jeffrey and D. Zwillinger, Academic Press, New York, 7th edition, 2007.

[17] E. W. Hobson, The Theory of Spherical and Ellipsoidal Harmonics, Cambridge University Press, Cambridge, UK, 1931.

[18] A. A. Kilbas, O. I. Marichev, S. G. Samko, Fractional integrals and derivatives : theory and applications, Gordon and Breach Science Publishers, Switzerland ; Philadelphia, Pa., USA, 1993.

[19] G. Kristensson, Second Order Differential Equations, Springer, New York, NY, 2010.

[20] A. Languasco, A. Zaccagnini, A Cesàro average of Goldbach numbers, Forum Math. 27(4) (2015), $1945-1960$.

[21] Y. Zhou, Legendre functions, spherical rotations, and multiple elliptic integrals. Ramanujan J. 34 (2014), 373-428.

Dipartimento di Ingegneria Industriale e Scienze Matematiche, Università Politecnica delle Marche, Via Brecce Bianche, 12, 60131 Ancona, Italia

Email address: m.cantarini@univpm.it 